\title{
Existence results for fractional neutral functional integro-differential evolution equations with infinite delay in Banach spaces
}

\author{
Chokkalingam Ravichandran ${ }^{1}$ and Dumitru Baleanu'2,3,4*
}

\author{
"Correspondence: \\ dumitru@cankaya.edu.tr \\ ${ }^{2}$ Department of Mathematics and \\ Computer Science, Faculty of Arts \\ and Sciences, Cankaya University, \\ Ankara, 06530, Turkey \\ ${ }^{3}$ Department of Chemical and \\ Materials Engineering, Faculty of \\ Engineering, King Abdulaziz \\ University, Jeddah, Saudi Arabia \\ Full list of author information is \\ available at the end of the article
}

\begin{abstract}
In this paper, we investigate the existence results for a class of abstract fractional neutral integro-differential evolution systems involving the Caputo derivative in Banach spaces. The main techniques rely on the fractional calculus, properties of characteristic solution operators, Mönch's fixed point theorem via measures of noncompactness. Particularly, we do not assume that characteristic solution operators are compact. The application is given to illustrate the theory. The results of this article are generalization and improvement of the recent results on this issue. MSC: 26A33; 34A12; 47H08; 47H10

Keywords: fractional Calculus; Caputo fractional derivative; existence; fixed point theorem; measures of noncompactness
\end{abstract}

\section{Introduction}

Fractional derivatives provide an excellent tool for the description of memory and hereditary properties of various materials and processes. For more details on fractional calculus theory and applications, one can see the monographs of Kilbas et al. [1], Lakshmikantham et al. [2], Miller and Ross [3], Podlubny [4], Baleanu et al. [5-7] and the papers [8-12] as well as the references therein.

Recently, the existence of solutions for fractional semilinear differential or integrodifferential equations is one of the theoretical fields investigated by many authors [11, 13-16]. Very recently, Ji et al. [17] studied the controllability of impulsive differential systems with nonlocal conditions by using Mönch's fixed point theorem and Wang et al. [18] established the sufficient conditions for nonlocal controllability for fractional evolution systems and the results were obtained by using fractional calculus and Mönch's fixed point theorem.

However, to the best of our knowledge, no work has been reported on the existence results for fractional neutral integro-differential systems with infinite delay in an abstract phase space via measures of noncompactness combined with the help of characteristic solution operators.

Our aim of this paper is to close the gap, and motivated by works $[11,17,18]$, in this paper we investigate the existence of solutions of fractional neutral integro-differential systems 
with infinite delay of the form

$$
\begin{aligned}
& { }^{C} D^{q}\left[x(t)-g\left(t, x_{t}\right)\right]=A x(t)+f\left(t, x_{t}, \int_{0}^{t} h\left(t, s, x_{s}\right) d s\right), \quad t \in J:=[0, b], \\
& x_{0}=\phi \in \mathcal{B}_{h}, \quad t \in(-\infty, 0]
\end{aligned}
$$

where ${ }^{C} D^{q}$ is the Caputo fractional derivative of order $0<q<1, A$ is the infinitesimal generator of a strongly continuous semigroup $\{T(t), t \geq 0\}$ in a Banach space $X$, which means that there exists $M_{1} \geq 1$ such that $\sup _{t \in J}\|T(t)\| \leq M_{1}, f: J \times \mathcal{B}_{h} \times X, g: J \times \mathcal{B}_{h}$ and $h: J \times J \times \mathcal{B}_{h}$ are given functions, where $\mathcal{B}_{h}$ is a phase space defined in Section 2. The histories $x_{t}:(-\infty, 0] \rightarrow X$, defined by $x_{t}(s)=x(t+s), s \leq 0$, belong to some abstract phase space $\mathcal{B}_{h}$.

The paper is organized as follows. In Section 2, we recall some basic definitions, notations and preliminary facts. Section 3 is devoted to the existence results for fractional neutral integro-differential evolution systems with infinite delay. The application of our theoretical results is given in Section 4. The last section is devoted to our conclusions.

\section{Preliminaries}

In this section, we mention notations, definitions, lemmas and preliminary facts needed to establish our main results.

Throughout this paper, we denote by $X$ a Banach space with the norm $\|\cdot\|$. Let $Y$ be another Banach space, let $L_{b}(X, Y)$ denote the space of bounded linear operators from $X$ to $Y$. We also use $\|f\|_{L^{p}\left(J, R^{+}\right)}$norm of $f$ whenever $f \in L^{p}\left(J, R^{+}\right)$for some $p$ with $1 \leq p \leq \infty$. Let $L^{p}(J, X)$ denote the Banach space of functions $f: J \times \mathcal{B}_{h} \times X \rightarrow X$ which are Bochner integrable normed by $\|f\|_{L^{p}(, X)}$. Let $\mathcal{C}(J, X)$, be the Banach space of continuous functions from $J$ into $X$ with the usual supremum norm $\|x\|_{\mathcal{C}}:=\sup _{t \in J}\|x(t)\|$, for $x \in \mathcal{C}$.

In this paper, we assume that $A: D(A) \subset X \rightarrow X$ is the infinitesimal generator of a strongly continuous semigroup $T(\cdot)$, then there exists a constant $M \leq 1$. Without loss of generality, we assume that $0 \in \rho(A)$. Then it is possible to define the fractional power $A^{\alpha}$ for $0<\alpha \leq 1$, as a closed linear operator on its domain $D\left(A^{\alpha}\right)$ with inverse $A^{-\alpha}$ (see [19]). The following are basic properties of $A^{\alpha}$.

(i) $D\left(A^{\alpha}\right)$ is a Banach space with the norm $\|x\|_{\alpha}=\left\|A^{\alpha} x\right\|$ for $x \in D\left(A^{\alpha}\right)$.

(ii) $T(t): X \rightarrow X_{\alpha}$ for $t \geq 0$.

(iii) $A^{\alpha} T(t) x=T(t) A^{\alpha} x$ for each $x \in D\left(A^{\alpha}\right)$ and $t \geq 0$.

(iv) For every $t>0, A^{\alpha} T(t)$ is bounded on $X$ and there exists $M_{\alpha}>0$ such that

$$
\left\|A^{\alpha} T(t)\right\| \leq \frac{M_{\alpha}}{t^{\alpha}}
$$

(v) $A^{-\alpha}$ is a bounded linear operator for $0 \leq \alpha \leq 1$ in $X$.

Now we define the abstract phase space $\mathcal{B}_{h}$, which has been used in [20]. Assume that $h:(-\infty, 0] \rightarrow(0,+\infty)$ is a continuous function with $l=\int_{-\infty}^{0} h(t) d t<+\infty$. For any $a>0$, we define

$$
\mathcal{B}=\{\psi:[-a, 0] \rightarrow X \text { such that } \psi(t) \text { is bounded and measurable }\}
$$


and equip the space $\mathcal{B}$ with the norm

$$
\|\psi\|_{[-a, 0]}=\sup _{s \in[-a, 0]}\|\psi(s)\|, \quad \forall \psi \in \mathcal{B} .
$$

Let us define

$$
\begin{aligned}
\mathcal{B}_{h}= & \left\{\psi:(-\infty, 0] \rightarrow X \text { such that for any } c>0,\left.\psi\right|_{[-c, 0]} \in \mathcal{B}\right. \\
& \text { and } \left.\int_{-\infty}^{0} h(s)\|\psi\|_{[s, 0]} d s<+\infty\right\} .
\end{aligned}
$$

If $\mathcal{B}_{h}$ is endowed with the norm

$$
\|\psi\|_{\mathcal{B}_{h}}=\int_{-\infty}^{0} h(s)\|\psi\|_{[s, 0]} d s, \quad \forall \psi \in \mathcal{B}_{h},
$$

then it is clear that $\left(\mathcal{B}_{h},\|\cdot\|_{\mathcal{B}_{h}}\right)$ is a Banach space.

Now we consider the space

$$
\mathcal{B}_{h}^{\prime}=\left\{x:(-\infty, b] \rightarrow X \text { such that }\left.x\right|_{J} \in \mathcal{C}(J, X), x_{0}=\phi \in \mathcal{B}_{h}\right\}
$$

Set $\|\cdot\|_{b}$ be a seminorm in $\mathcal{B}_{h}^{\prime}$ defined by

$$
\|x\|_{b}=\|\phi\|_{\mathcal{B}_{h}}+\sup \{\|x(s)\|: s \in[0, b]\}, \quad x \in \mathcal{B}_{h}^{\prime} .
$$

Lemma 2.1 (See [20]) Assume $x \in \mathcal{B}_{h}^{\prime}$, then for $t \in J, x_{t} \in \mathcal{B}_{h}$. Moreover,

$$
l|x(t)| \leq\left\|x_{t}\right\|_{\mathcal{B}_{h}} \leq\|\phi\|_{\mathcal{B}_{h}}+l \sup _{s \in[0, t]}|x(s)|,
$$

where $l=\int_{-\infty}^{0} h(t) d t<+\infty$.

Let us recall the following known definitions. For more details see $[1,3,6]$.

Definition 2.1 The fractional integral of order $\alpha$ with the lower limit zero for a function $f$ is defined as

$$
I^{\alpha} f(t)=\frac{1}{\Gamma(\alpha)} \int_{0}^{t} \frac{f(s)}{(t-s)^{1-\alpha}} d s, \quad t>0, \alpha>0,
$$

provided the right-hand side is pointwise defined on $[0, \infty)$, where $\Gamma(\cdot)$ is the gamma function, which is defined by $\Gamma(\alpha)=\int_{0}^{\infty} t^{\alpha-1} e^{-t} d t$.

Definition 2.2 The Riemann-Liouville fractional derivative of order $\alpha>0, n-1<\alpha<n$, $n \in N$, is defined as

$$
{ }^{(R-L)} D_{0+}^{\alpha} f(t)=\frac{1}{\Gamma(n-\alpha)}\left(\frac{d}{d t}\right)^{n} \int_{0}^{t}(t-s)^{n-\alpha-1} f(s) d s,
$$

where the function $f(t)$ has absolutely continuous derivative up to order $(n-1)$. 
Definition 2.3 The Caputo derivative of order $\alpha$ for a function $f:[0, \infty) \rightarrow R$ can be written as

$$
D^{\alpha} f(t)=D^{\alpha}\left(f(t)-\sum_{k=0}^{n-1} \frac{t^{k}}{k !} f^{(k)}(0)\right), \quad t>0, n-1<\alpha<n .
$$

\section{Remark 2.1}

(i) If $f(t) \in C^{n}[0, \infty)$, then

$$
{ }^{C} D^{\alpha} f(t)=\frac{1}{\Gamma(n-\alpha)} \int_{0}^{t} \frac{f^{(n)}(s)}{(t-s)^{\alpha+1-n}} d s=I^{n-\alpha} f^{(n)}(t), \quad t>0, n-1<\alpha<n .
$$

(ii) The Caputo derivative of a constant is equal to zero.

(iii) If $f$ is an abstract function with values in $X$, then integrals which appear in Definitions 2.1 and 2.2 are taken in Bochner's sense.

Definition 2.4 (See [11]) A continuous function $x:(-\infty, b] \rightarrow X$ is said to be a mild solution of system (1.1)-(1.2) if $x_{0}=\phi \in \mathcal{B}_{h}$ on $(-\infty, 0]$ and the following integral equation

$$
\begin{aligned}
x(t)= & \mathcal{T}(t)[\phi(0)-g(0, \phi(0))]+g\left(t, x_{t}\right)+\int_{0}^{t}(t-s)^{q-1} A \mathcal{S}(t-s) g\left(s, x_{s}\right) d s \\
& +\int_{0}^{t}(t-s)^{q-1} \mathcal{S}(t-s) f\left(s, x_{s}, \int_{0}^{s} h\left(s, \tau, x_{\tau}\right) d \tau\right) d s, \quad t \in J,
\end{aligned}
$$

is satisfied, where $\mathcal{T}(\cdot)$ and $\mathcal{S}(\cdot)$ are called characteristic solution operators and given by

$$
\mathcal{T}=\int_{0}^{\infty} \xi_{q}(\theta) T\left(t^{q} \theta\right) d \theta, \quad \mathcal{S}=q \int_{0}^{\infty} \theta \xi_{q}(\theta) T\left(t^{q} \theta\right) d \theta
$$

and for $\theta \in(0, \infty)$

$$
\begin{aligned}
& \xi_{q}(\theta)=\frac{1}{q} \theta^{-1-\frac{1}{q}} \bar{w}_{q}\left(\theta^{-\frac{1}{q}}\right) \geq 0, \\
& \bar{w}_{q}(\theta)=\frac{1}{\pi} \sum_{n=1}^{\infty}(-1)^{n-1} \theta^{-n q-1} \frac{\Gamma(n q+1)}{n !} \sin (n \pi q) .
\end{aligned}
$$

Here, $\xi_{q}$ is a probability density function defined on $(0, \infty)$, that is

$$
\xi_{q}(\theta) \geq 0, \quad \theta \in(0, \infty) \quad \text { and } \quad \int_{0}^{\infty} \xi_{q}(\theta) d \theta=1
$$

The following results of $\mathcal{T}(\cdot)$ and $\mathcal{S}(\cdot)$ are used throughout this paper.

Remark 2.2 (See [11]) It is not difficult to verify that for $v \in[0,1]$,

$$
\int_{0}^{\infty} \theta^{v} \xi_{q}(\theta) d \theta=\int_{0}^{\infty} \theta^{-q v} \bar{w}_{q}(\theta) d \theta=\frac{\Gamma(1+v)}{\Gamma(1+q v)} .
$$

Lemma 2.2 (See $[11,18])$ The operators $\mathcal{T}$ and $\mathcal{S}$ have the following properties: 
(i) For any fixed $t \geq 0, \mathcal{T}$ and $\mathcal{S}$ are linear and bounded operators, that is, for any $x \in X$,

$$
\|\mathcal{T}(t) x\| \leq M\|x\| \quad \text { and } \quad\|\mathcal{S}(t) x\| \leq \frac{q M}{\Gamma(1+q)}\|x\| .
$$

(ii) $\{\mathcal{T}(t), t \geq 0\}$ and $\{\mathcal{S}(t), t \geq 0\}$ are strongly continuous.

(iii) For $t \in J$ and any bounded subsets $D \subset X, t \rightarrow\{\mathcal{T}(t) x: x \in D\}$ and $t \rightarrow\{\mathcal{S}(t) x: x \in D\}$ are equicontinuous if $\left\|T\left(t_{2}^{q}(\theta)\right) x-T\left(t_{1}^{q}(\theta)\right) x\right\| \rightarrow 0$ with respect to $x \in D$ as $t_{2} \rightarrow t_{1}$ for each fixed $\theta \in[0, \infty]$.

(iv) For any $x \in X, \alpha, \beta \in(0,1)$, we have

$$
\begin{aligned}
& A T_{q}(t) x=A^{1-\beta} T_{q}(t) A^{\beta} x, \quad t \in J, \\
& \left\|A^{\alpha} T_{q}(t)\right\| \leq \frac{q M_{\alpha} \Gamma(2-\alpha)}{\Gamma(1+q(1-\alpha))} t^{-\alpha q}, \quad 0<t \leq b .
\end{aligned}
$$

Moreover, let us recall some definitions and properties of the measures of noncompactness.

Definition 2.5 (See [17]) Let $E^{+}$be a positive cone of an ordered Banach space $(E, \leq)$. A function $\Phi$ defined on the set of all bounded subsets of the Banach space $X$ with values in $E^{+}$is called a measure of noncompactness (MNC) on $X$ iff $\Phi(\overline{c o} \Omega)=\Phi(\Omega)$ for all bounded subsets $\Omega \subseteq X$, where $\overline{c o} \Omega$ stands for the closed convex hull of $\Omega$.

The MNC $\Phi$ is said to be

(1) Monotone iff for all bounded subsets $\Omega_{1}, \Omega_{2}$ of $X$ we have:

$$
\left(\Omega_{1} \subseteq \Omega_{2}\right) \quad \Rightarrow \quad\left(\Phi\left(\Omega_{1}\right) \leq \Phi\left(\Omega_{2}\right)\right)
$$

(2) Nonsingular iff $\Phi(\{a\} \cup \Omega)=\Phi(\Omega)$ for every $a \in X, \Omega \subset X$;

(3) Regular iff $\Phi(\Omega)=0$ if and only if $\Omega$ is relatively compact in $X$.

One of the many examples of MNC is the noncompactness measure of Hausdorff $\beta$ defined on each bounded subset $\Omega$ of $X$ by

$$
\begin{aligned}
\beta(\Omega)= & \inf \{\epsilon>0 ; \Omega \text { can be covered by a finite number of balls of radii } \\
& \text { smaller than } \epsilon\} .
\end{aligned}
$$

It is well known that MNC $\beta$ enjoys the above properties and other properties see [21, 24]. For all bounded subsets $\Omega, \Omega_{1}, \Omega_{2}$ of $X$,

(4) $\beta\left(\Omega_{1}+\Omega_{2}\right) \leq \beta\left(\Omega_{1}\right)+\beta\left(\Omega_{2}\right)$, where $\Omega_{1}+\Omega_{2}=\left\{x+y: x \in \Omega_{1}, y \in \Omega_{2}\right\}$;

(5) $\beta\left(\Omega_{1} \cup \Omega_{2}\right) \leq \max \left\{\beta\left(\Omega_{1}\right), \beta\left(\Omega_{2}\right)\right\}$;

(6) $\beta(\lambda \Omega) \leq|\lambda| \beta(\Omega)$ for any $\lambda \in R$;

(7) If the map $Q: D(Q) \subseteq X \rightarrow Z$ is Lipschitz continuous with constant $k$, then $\beta_{Z}(Q \Omega) \leq k \beta(\Omega)$ for any bounded subset $\Omega \subseteq D(Q)$, where $Z$ is a Banach space.

Lemma 2.3 (See [24]) If $W \subset C([a, b], X)$ is bounded and equicontinuous, then $\beta(W(t))$ is continuous for $t \in[a, b]$ and

$$
\beta(W)=\sup \{\beta(W(t)), t \in[a, b]\}, \quad \text { where } W(t)=\{x(t): x \in W\} \subseteq X .
$$


Theorem 2.1 (See $[18,22])$ If $\left\{u_{n}\right\}_{n=1}^{\infty}$ is a sequence of Bochner integrable functions from $J$ into $X$ with the estimation $\left\|u_{n}(t)\right\| \leq \mu(t)$ for almost all $t \in J$ and every $n \geq 1$, where $\mu \in L^{1}(J, R)$, then the function $\psi(t)=\beta\left(\left\{u_{n}(t): n \geq 1\right\}\right)$ belongs to $L^{1}(J, R)$ and satisfies $\beta\left(\left\{\int_{0}^{t} \psi(s) d s: n \geq 1\right\}\right) \leq 2 \int_{0}^{t} \psi(s) d s$.

The following fixed-point theorem, a nonlinear alternative of Mönch's type, plays a key role in our proof of system (1.1)-(1.2).

Lemma 2.4 (See [23, Theorem 2.2]) Let D be a closed convex subset of a Banach space $X$ and $0 \in D$. Assume that $F: D \rightarrow X$ is a continuous map which satisfies Mönch's condition, that is, $(M \subseteq D$ is countable, $M \subseteq \overline{c o}(\{0\} \cup F(M)) \Rightarrow \bar{M}$ is compact $)$. Then $F$ has a fixed point in $D$.

\section{Existence results}

In this section, we present and prove the existence results for problem (1.1)-(1.2). In order to prove the main theorem of this section, we list the following hypotheses.

(H1) (i) $A$ generates a strongly continuous semigroup $\{T(t): t \geq 0\}$ in $X$;

(ii) For all bounded subsets $D \subset X$ and $x \in D,\left\|T\left(t_{2}^{q} \theta\right) x-T\left(t_{1}^{q} \theta\right) x\right\| \rightarrow 0$ as $t_{2} \rightarrow t_{1}$ for each fixed $\theta \in(0, \infty)$.

(H2) The function $f: J \times \mathcal{B}_{h} \times X \rightarrow X$ satisfies the following:

(i) $f(\cdot, \phi, x)$ is measurable for all $(\phi, x) \in \mathcal{B}_{h} \times X$ and $f(t, \cdot, \cdot)$ is continuous for a.e. $t \in J$ and for $x \in \mathcal{B}_{h}, f(\cdot, \cdot, x):[0, T] \rightarrow X$ is strongly measurable.

(ii) There exists a constant $q_{1} \in(0, q)$ and $m \in L^{\frac{1}{q_{1}}}\left(J, R^{+}\right)$and a nondecreasing continuous function, there is a positive integrable function $\Omega: R^{+} \rightarrow R^{+}$such that $\|f(t, \phi, x)\| \leq m(t) \Omega\left(\|\phi\|_{\mathcal{B}_{h}}+\|x\|\right)$, for all $(t, \phi, x) \in J \times \mathcal{B}_{h} \times X$, where $\Omega$ satisfies $\liminf _{n \rightarrow \infty} \frac{\Omega(n)}{n}=0$.

(iii) There exists a constant $q_{2} \in(0, q)$ and $h \in L^{\frac{1}{q_{2}}}\left(J, R^{+}\right)$such that, for any bounded subset $D_{1} \subset X, F_{1} \subset \mathcal{B}_{h}$,

$$
\beta\left(f\left(t, F_{1}, D_{1}\right)\right) \leq \eta(t)\left[\sup _{-\infty<\theta \leq 0} \beta\left(F_{1}(\theta)\right)+\beta\left(D_{1}\right)\right] \text { for a.e. } t \in J,
$$

where $F_{1}(\theta)=\left\{v(\theta): v \in D_{1}\right\}$ and $\beta$ is the Hausdorff MNC.

(H3) The function $h: J \times J \times \mathcal{B}_{h} \rightarrow X$ satisfies:

(i) $h(\cdot, \phi, x)$ is measurable for $(\phi, x) \in \mathcal{B}_{h} \times X$ and $h(t, \cdot, \cdot)$ is continuous for a.e. $t \in J$.

(ii) There exists a constant $H_{0}>0$ such that $\|h(t, s, \phi)\| \leq H_{0}\left(1+\|\phi\|_{\mathcal{B}_{h}}\right)$, for all $t, s \in J, \phi \in \mathcal{B}_{h}$.

(iii) There exists $\zeta \in L^{1}\left(J^{2}, R^{+}\right)$such that for any bounded subset $D_{2} \subset X$,

$$
\beta\left(h\left(t, s, D_{2}\right)\right) \leq \zeta(t, s)\left[\sup _{-\infty<\theta \leq 0} \beta\left(D_{2}(\theta)\right)\right] \text { for a.e. } t \in J
$$

with $\zeta^{*}=\sup _{s \in J} \int_{0}^{s} \zeta(t, \tau) d \tau<\infty$.

(H4) The function $g: J \times \mathcal{B}_{h}$ is continuous and there exists a constant $H_{1}>0,0<\alpha<1$ such that $g$ is $X_{\alpha}$ valued and

$$
\begin{aligned}
& \left\|A^{\beta} g(t, x)-A^{\beta} g(t, y)\right\| \leq H_{1}\|x-y\|_{\mathcal{B}_{h}}, \quad x, y \in \mathcal{B}_{h}, t \in J:=[0, b], \\
& \left\|A^{\beta} g(t, x)\right\| \leq H_{1}\left(1+\|x\|_{\mathcal{B}_{h}}\right) .
\end{aligned}
$$


For our convenience, let us take $\kappa_{i}:=\left[\left(\frac{1-q_{i}}{q-q_{i}}\right) b^{\frac{q-q_{i}}{1-q_{i}}}\right]^{1-q_{i}}, i=0,1,2 . M_{4}:=\kappa_{1}\|m\|_{L^{\frac{1}{q_{1}}}\left(J, R^{+}\right)}$, and $M_{6}=\kappa_{2}\|\eta\|_{L^{\frac{1}{q_{2}}}\left(J, R^{+}\right)}$.

Theorem 3.1 Assume that the hypotheses (H1)-(H4) are satisfied, then system (1.1)-(1.2) has at least one mild solution provided that,

$$
Z^{*}=\frac{2 M_{1} M_{6} q}{\Gamma(1+q)}\left(1+2 \zeta^{*}\right)<1 \quad \text { for some } \frac{1}{2}<q<1 .
$$

Proof In order to prove the existence of mild solutions for system (1.1)-(1.2), transform it into a fixed point problem.

We consider the operator $\Phi: \mathcal{B}_{h}^{\prime} \rightarrow \mathcal{B}_{h}^{\prime}$ defined by

$$
\Phi x(t)=\left\{\begin{array}{l}
\phi(t), \quad t \in(-\infty, 0], \\
\mathcal{T}(t)[\phi(0)-g(0, \phi(0))]+g\left(t, x_{t}\right)+\int_{0}^{t}(t-s)^{q-1} A \mathcal{S}(t-s) g\left(s, x_{s}\right) d s \\
\quad+\int_{0}^{t}(t-s)^{q-1} \mathcal{S}(t-s) f\left(s, x_{s}, \int_{0}^{s} h\left(s, \tau, x_{\tau}\right) d \tau\right) d s, \quad t \in J .
\end{array}\right.
$$

For $\phi \in \mathcal{B}_{h}$, we define $\hat{\phi}$ by

$$
\hat{\phi}(t)= \begin{cases}\phi(t), & t \in(-\infty, 0] \\ \mathcal{T}(t) \phi(0), & t \in J\end{cases}
$$

then $\hat{\phi} \in \mathcal{B}_{h}^{\prime}$. Let $x(t)=y(t)+\hat{\phi}(t),-\infty<t \leq b$. It is easy to see that $x$ satisfies (2.1) if and only if $y$ satisfies $y_{0}=0$ and

$$
\begin{aligned}
y(t)= & -\mathcal{T}(t) g(0, \phi)+g\left(t, y_{t}+\hat{\phi}_{t}\right)+\int_{0}^{t}(t-s)^{q-1} A \mathcal{S}(t-s) g\left(s, y_{s}+\hat{\phi}_{s}\right) d s \\
& +\int_{0}^{t}(t-s)^{q-1} \mathcal{S}(t-s) f\left(s, y_{s}+\hat{\phi}_{s}, \int_{0}^{s} h\left(s, \tau, y_{\tau}+\hat{\phi}_{\tau}\right) d \tau\right) d s .
\end{aligned}
$$

Let $\mathcal{B}_{h}^{\prime \prime}=\left\{y \in \mathcal{B}_{h}^{\prime}: y_{0}=0 \in \mathcal{B}_{h}\right\}$. For any $y \in \mathcal{B}_{h}^{\prime \prime}$,

$$
\begin{aligned}
\|y\|_{b} & =\left\|y_{0}\right\|_{\mathcal{B}_{h}}+\sup \{\|y(s)\|: 0 \leq s \leq b\} \\
& =\sup \{\|y(s)\|: 0 \leq s \leq b\},
\end{aligned}
$$

thus $\left(\mathcal{B}_{h}^{\prime \prime},\|\cdot\|_{b}\right)$ is a Banach space. Set $B_{r}=\left\{y \in \mathcal{B}_{h}^{\prime \prime}:\|y\|_{b} \leq r\right\}$ for some $r>0$, then $B_{r} \subseteq \mathcal{B}_{h}^{\prime \prime}$ is uniformly bounded, and for $y \in B_{r}$, from Lemma 2.1, we have

$$
\begin{aligned}
\left\|y_{t}+\hat{\phi}_{t}\right\|_{\mathcal{B}_{h}} & \leq\left\|y_{t}\right\|_{\mathcal{B}_{h}}+\left\|\hat{\phi}_{t}\right\|_{\mathcal{B}_{h}} \\
& \leq l\left(r+M_{1}|\phi(0)|\right)+\|\phi\|_{\mathcal{B}_{h}}=r^{\prime} .
\end{aligned}
$$

Define the operator $\widetilde{\Phi}: \mathcal{B}_{h}^{\prime \prime} \rightarrow \mathcal{B}_{h}^{\prime \prime}$ by

$$
\widetilde{\Phi} y(t)=\left\{\begin{array}{l}
0, \quad t \in(-\infty, 0], \\
-\mathcal{T}(t) g(0, \phi)+g\left(t, y_{t}+\hat{\phi}_{t}\right)+\int_{0}^{t}(t-s)^{q-1} A \mathcal{S}(t-s) g\left(s, y_{s}+\hat{\phi}_{s}\right) d s \\
\quad+\int_{0}^{t}(t-s)^{q-1} \mathcal{S}(t-s) f\left(s, y_{s}+\hat{\phi}_{s}, \int_{0}^{s} h\left(s, \tau, y_{\tau}+\hat{\phi}_{\tau}\right) d \tau\right) d s, \quad t \in J .
\end{array}\right.
$$


Obviously, the operator $\Phi$ has a fixed point is equivalent to $\widetilde{\Phi}$ has one. So, our aim is to show that $\widetilde{\Phi}$ has a fixed point. The proof will be given in several steps.

Step 1: We show that there exists some $r>0$ such that $\widetilde{\Phi}\left(B_{r}\right) \subseteq B_{r}$. If it is not true, then for each positive number $r$, there exists a function $y^{r}(\cdot) \in B_{r}$ and some $t \in J$ such that $\left\|\left(\widetilde{\Phi} y^{r}\right)(t)\right\|>r$.

On the other hand, from hypotheses the (H2)(i), (ii), (H4), Lemma 2.2(i) and Hölder's inequality, we obtain

$$
\begin{aligned}
r< & \left\|\left(\widetilde{\Phi} y^{r}\right)(t)\right\| \\
\leq & \|-\mathcal{T}(t) g(0, \phi)\|+\left\|g\left(t, y_{t}+\hat{\phi}_{t}\right)\right\|+\left\|\int_{0}^{t}(t-s)^{q-1} A \mathcal{S}(t-s) g\left(s, y_{s}+\hat{\phi}_{s}\right) d s\right\| \\
& +\left\|\int_{0}^{t}(t-s)^{q-1} \mathcal{S}(t-s) f\left(s, y_{s}+\hat{\phi}_{s}, \int_{0}^{s} h\left(s, \tau, y_{\tau}+\hat{\phi}_{\tau}\right) d \tau\right) d s\right\| \\
:= & I_{1}+I_{2}+I_{3}+I_{4} .
\end{aligned}
$$

Let us estimate, $I_{i}, i=1,2,3,4$. By assumption (H4), we have

$$
\begin{aligned}
I_{1} & \leq M_{1}\left\|A^{-\beta}\right\|\left\|A^{\beta} g(0, \phi)\right\| \\
& \leq M_{1} H_{1}\left\|A^{-\beta}\right\|\left(1+\|\phi\|_{\mathcal{B}_{h}}\right), \\
I_{2} & \leq\left\|A^{-\beta}\right\|\left\|A^{\beta} g\left(t, y_{t}+\hat{\phi}_{t}\right)\right\| \\
& \leq H_{1}\left\|A^{-\beta}\right\|\left(1+\left\|y_{t}+\hat{\phi}_{t}\right\|_{\mathcal{B}_{h}}\right) \\
& \leq H_{1}\left\|A^{-\beta}\right\|\left(1+r^{\prime}\right) .
\end{aligned}
$$

By using Lemma 2.2 and Hölder's inequality, one can deduce that

$$
\begin{aligned}
I_{3} & \leq \int_{0}^{t}\left\|(t-s)^{q-1} A^{1-\beta} \mathcal{S}(t-s) A^{\beta} g\left(s, y_{s}+\hat{\phi}_{s}\right)\right\| d s \\
& \leq \frac{M_{1-\beta} \alpha \Gamma(1+\beta)}{\Gamma(1+\alpha \beta)} \int_{0}^{t}(t-s)^{\alpha \beta-1}\left\|A^{\beta} g\left(s, y_{s}+\hat{\phi}_{s}\right)\right\| d s \\
& \leq K_{1}(\alpha, \beta) \int_{0}^{t}(t-s)^{\alpha \beta-1} H_{1}\left(1+\|x\|_{\mathcal{B}_{h}}\right) d s \\
& \leq K_{1}(\alpha, \beta) H_{1} \frac{T^{\alpha \beta}}{\alpha \beta}\left(1+r^{\prime}\right) .
\end{aligned}
$$

Using assumptions (H2) and (H3), we have

$$
\begin{aligned}
I_{4} & \leq\left\|\int_{0}^{t}(t-s)^{q-1} \mathcal{S}(t-s) f\left(s, y_{s}+\hat{\phi}_{s}, \int_{0}^{s} h\left(s, \tau, y_{\tau}+\hat{\phi}_{\tau}\right)\right) d \tau\right\| d s \\
& \leq \frac{M_{1}}{\Gamma(\alpha)} \int_{0}^{t}(t-s)^{q-1} m(s) \Omega\left(\|\phi\|_{\mathcal{B}_{h}}+\|x\|\right) d s \\
& \leq \frac{M_{1} T^{\alpha}}{\Gamma(\alpha+1)} m(s) \Omega\left(r^{\prime}+b H_{0}\left(1+r^{\prime}\right)\right) d s \\
& \leq \frac{M_{1} T^{\alpha}}{\Gamma(\alpha+1)} \Omega\left(r^{\prime}+b H_{0}\left(1+r^{\prime}\right)\right) \sup _{t \in J} m(s) .
\end{aligned}
$$


Combining estimates (3.5)-(3.8) yields

$$
\begin{aligned}
I_{1}+I_{2}+I_{3}+I_{4}< & H_{1}\left\|A^{-\beta}\right\|\left[M_{1}\left(1+\|\phi\|_{\mathcal{B}_{h}}\right)+\left(1+r^{\prime}\right)\right]+K(\alpha, \beta) H_{1} \frac{T^{\alpha \beta}}{\alpha \beta}\left(1+r^{\prime}\right) \\
& +\frac{M_{1} T^{\alpha}}{\Gamma(\alpha+1)} \Omega\left(r^{\prime}+b H_{0}\left(1+r^{\prime}\right)\right) \sup _{t \in J} m(s) .
\end{aligned}
$$

Dividing both sides of (3.9) by $r$, and taking $r \rightarrow \infty$, we have $\geq 1$, which is a contradiction. Hence for some positive number $r, \widetilde{\Phi}\left(B_{r}\right) \subseteq B_{r}$.

Step 2: $\widetilde{\Phi}$ is continuous on $B_{r}$.

Let $\left\{y^{n}\right\}_{n \in N} \subset B_{r}$ with $y^{(n)} \rightarrow y$ in $B_{r}$ as $n \rightarrow \infty$.

Denote

$$
\begin{aligned}
& F_{n}(s)=f\left(s, y_{s}^{(n)}+\hat{\phi}_{s}, \int_{0}^{s} h\left(s, \tau, y_{\tau}^{(n)}+\hat{\phi}_{\tau}\right) d \tau\right) \text { and } \\
& F(s)=f\left(s, y_{s}+\hat{\phi}_{s}, \int_{0}^{s} h\left(s, \tau, y_{\tau}+\hat{\phi}_{\tau}\right) d \tau\right) .
\end{aligned}
$$

Then, by using hypotheses $\left(\mathrm{H}_{2}\right)(\mathrm{i})$, (ii), $\left(\mathrm{H}_{3}\right)(\mathrm{i})$, (ii) and Lebesgue's dominated convergence theorem, we obtain

$$
\int_{0}^{t}(t-s)^{q-1}\left\|F_{n}(s)-F(s)\right\| d s \rightarrow 0 \quad \text { as } n \rightarrow \infty, t \in J
$$

Now,

$$
\left\|\widetilde{\Phi} y^{n}-\widetilde{\Phi} y\right\|_{\mathcal{C}} \leq \frac{M_{1} q}{\Gamma(1+q)} \int_{0}^{t}(t-s)^{q-1}\left\|F_{n}(s)-F(s)\right\| d s .
$$

Observing (3.6) and (3.7), we have

$$
\left\|\widetilde{\Phi} y^{n}-\widetilde{\Phi} y\right\|_{\mathcal{C}} \rightarrow 0 \quad \text { as } n \rightarrow \infty
$$

which implies that $\widetilde{\Phi}$ is continuous on $B_{r}$.

Step 3: $\widetilde{\Phi}\left(B_{r}\right)$ is equicontinuous on $J$. Indeed, let $z \in \widetilde{\Phi}\left(B_{r}\right)$ and $0 \leq t_{1}<t_{2} \leq b$. Then there is $y \in B_{r}$ such that

$$
\begin{aligned}
& \left\|z\left(t_{2}\right)-z\left(t_{1}\right)\right\| \\
& \leq\left\|\mathcal{T}(t)\left(t_{2}\right)-\mathcal{T}(t)\left(t_{1}\right)\right\|+[\|\phi(0)\|+\| g(0, \phi(0) \|] \\
& \quad+\left\|\int_{0}^{t_{2}}\left(t_{2}-s\right)^{q-1} \mathcal{S}\left(t_{2}-s\right) F(s) d s-\int_{0}^{t_{1}}\left(t_{1}-s\right)^{q-1} \mathcal{S}\left(t_{1}-s\right) F(s) d s\right\| \\
& \leq\left\|\mathcal{T}(t)\left(t_{2}\right)-\mathcal{T}(t)\left(t_{1}\right)\right\|+\left[\phi+\|g(0, \phi(0) \|]+\| \int_{t_{1}}^{t_{2}}\left(t_{2}-s\right)^{q-1} \mathcal{S}\left(t_{2}-s\right) F(s) d s \|\right. \\
& \quad+\left\|\int_{t_{1}-\epsilon}^{t_{1}}\left(t_{2}-s\right)^{q-1}\left[\mathcal{S}\left(t_{2}-s\right)-\mathcal{S}\left(t_{1}-s\right)\right] F(s) d s\right\| \\
& \quad+\left\|\int_{t_{1}-\epsilon}^{t_{1}}\left[\left(t_{2}-s\right)^{q-1}-\left(t_{1}-s\right)^{q-1}\right] \mathcal{S}\left(t_{1}-s\right) F(s) d s\right\|
\end{aligned}
$$




$$
\begin{aligned}
& +\left\|\int_{0}^{t_{1}-\epsilon}\left(t_{2}-s\right)^{q-1}\left[\mathcal{S}\left(t_{2}-s\right)-\mathcal{S}\left(t_{1}-s\right)\right] F(s) d s\right\| \\
& +\left\|\int_{0}^{t_{1}-\epsilon}\left[\left(t_{2}-s\right)^{q-1}-\left(t_{1}-s\right)^{q-1}\right] \mathcal{S}\left(t_{1}-s\right) F(s) d s\right\| .
\end{aligned}
$$

Using Lemma 2.2, we can verify that the right-hand side of the above inequality tends to zero as $t_{2} \rightarrow t_{1}$. Therefore, $\widetilde{\Phi}\left(B_{r}\right)$ is equicontinuous on $J$.

Step 4: Mönch's condition holds.

Suppose that $W \subseteq B_{r}$ is countable and $W \subseteq \operatorname{conv}(\{0\} \cup \widetilde{\Phi}(W))$. We show that $\beta(W)=0$, where $\beta$ is the Hausdorff MNC. Without loss of generality, we may suppose that $W=$ $\left\{y^{n}\right\}_{n=1}^{\infty}$. Now we need to show that $\widetilde{\Phi}(W)(t)$ is relatively compact in $X$ for each $t \in J$.

By Theorem 2.1, we have

$$
\begin{aligned}
& \beta\left(\left\{\widetilde{\Phi}_{y^{n}}(s)\right\}_{n=1}^{\infty}\right) \\
& \leq \beta\left(\left\{\int_{0}^{t}(t-s)^{q-1} \mathcal{S}(t-s) F_{n}(s) d s\right\}_{n=1}^{\infty}\right) \\
& \leq \frac{2 M_{1} q}{\Gamma(1+q)} \int_{0}^{t}(t-s)^{q-1} \beta\left(\left\{F_{n}(s) d s\right\}_{n=1}^{\infty}\right) d s \\
& \leq \frac{2 M_{1} q}{\Gamma(1+q)} \int_{0}^{t}(t-s)^{q-1} \eta(s)\left[\sup _{-\infty<\theta \leq 0} \beta\left(\left\{y^{n}(s+\theta)+\hat{\phi}(s+\theta)\right\}_{n=1}^{\infty}\right)\right. \\
&\left.+\beta\left(\left\{\int_{0}^{s} h\left(s, \tau, y_{\tau}^{n}+\hat{\phi}_{\tau}\right) d \tau\right\}_{n=1}^{\infty}\right)\right] d s \\
& \leq {\left[\frac{2 M_{1} q}{\Gamma(1+q)}\left(1+2 \zeta^{*}\right) M_{6}\right] \sup _{0 \leq \tau \leq s} \beta(W(\tau)) d s . }
\end{aligned}
$$

That is,

$$
\beta(\widetilde{\Phi} W(t)) \leq \frac{2 M_{1} M_{6} q}{\Gamma(1+q)}\left(1+2 \zeta^{*}\right) \sup _{0 \leq \tau \leq s} \beta(W(\tau))
$$

which implies, by Lemma $2.3, \beta(\widetilde{\Phi}(W)) \leq Z^{*} \beta(W)$, where $Z^{*}$ is defined in condition (3.1). Thus, from Mönch's condition, we get

$$
\beta(W) \leq \beta(\operatorname{conv}(\{0\} \cup \widetilde{\Phi}(W)))=\beta(\widetilde{\Phi}(W)) \leq Z^{*} \beta(W),
$$

which implies that $\beta(W)=0$.

Hence, using Lemma 2.4, $\widetilde{\Phi}$ has a fixed point $y$ in $B_{r}$. Then $x=y+\hat{\phi}$ is a mild solution of system (1.1)-(1.2). This completes the proof.

\section{An application}

For $1 / 2<\alpha<1$, consider the following fractional order neutral functional integrodifferential of the form

$$
\begin{aligned}
{ }^{C} D_{t}^{q}\left[z(t, \eta)+\int_{0}^{\pi} b(\theta, \eta) z(t, \theta) d \theta\right] \\
=\frac{\partial^{2}}{\partial \eta^{2}} z(t, \eta)
\end{aligned}
$$




$$
\begin{aligned}
& +\mu\left(t, \int_{-\infty}^{t} \mu_{1}(s-t) z(s, \eta) d s, \int_{0}^{t} \int_{-\infty}^{0} \mu_{2}(s, \eta, \tau-s) z(\tau, \eta) d \tau d s\right), \\
& z(t, 0)=z(t, \pi)=0, \quad t \geq 0 \\
& z(0, \eta)=\psi(\eta), \quad 0 \leq \eta \leq \pi
\end{aligned}
$$

where $\mu: J \times[0,1] \times[0,1] \times R \rightarrow R$ is continuous functions. To write system (1.1)-(1.3) to the form (4.1)-(4.3), let $X=L^{2}[0, \pi]$ and $A: D(A) \subset X \rightarrow X$ be defined as follows: $A f=f^{\prime}$ with domain

$$
D(A)=\left\{f \in X: f, f^{\prime} \text { are absolutely continuous, } f^{\prime \prime} \in X, f(0)=f(\pi)=0\right\} .
$$

It is well known that $A$ is an infinitesimal generator of a semigroup $\{T(t), t \geq 0\}$ in $X$ and is given by $T(t) w(s)=w(t+s)$, for $w \in X, T(t)$ is not a compact semigroup on $X$ with $\beta(T(t) D) \leq \beta(D)$, where $\beta$ is the Hausdorff MNC and there exists an $M_{1} \geq 1$ such that $\sup _{t \in J}\|T(t)\| \leq M_{1}$. Moreover, $t \rightarrow w\left(t^{\alpha} \theta+s\right) x$ is equicontinuous [18] for $t \geq 0$ and $\theta \in$ $(0, \infty)$. Define $f, g:[0, \pi] \times X \rightarrow X$ by

$$
\begin{aligned}
& g(z)(\eta)=\int_{0}^{\pi} b(\theta, \eta) z(\theta) d \theta, \\
& f\left(t, \pi, \int_{0}^{t} g(s, \pi) d s\right)(\eta) \\
& \quad=\mu\left(t, \int_{-\infty}^{t} \mu_{1}(s-t) z(s, \eta) d s, \int_{0}^{t} \int_{-\infty}^{0} \mu_{2}(s, \eta, \tau-s) z(\tau, \eta) d \tau d s\right) .
\end{aligned}
$$

We take $\mu\left(t, \int_{-\infty}^{t} \mu_{1}(s-t) z(s, \eta) d s, \int_{0}^{t} \int_{-\infty}^{0} \mu_{2}(s, \eta, \tau-s) z(\tau, \eta) d \tau d s\right)=C_{0} \sin (x(s)), C_{0}$ is a constant. $F$ is Lipschitz continuous for the second variable. Then $f$ satisfies hypotheses (H3) and (H4). This completes the example.

\section{Conclusions}

In the current paper, we are focused on establishing the existence result for a class of abstract fractional neutral functional integro-differential evolution systems involving the Caputo fractional derivative in Banach spaces. By using fractional calculus, the properties of characteristic solution operators, Mönch's fixed point theorem via MNC, we have found the existence results. Here, we do not assume that characteristic solution operators are compact. An example is provided to show the effectiveness of the proposed results.

\section{Competing interests}

The authors declare that they have no competing interests.

\section{Authors' contributions}

The authors have made equal contributions of this manuscript. All authors read and approved the final version.

\section{Author details}

'Department of Mathematics, RVS Faculty of Engineering, RVS Technical Campus, Coimbatore, Tamil Nadu 641402 , India. ${ }^{2}$ Department of Mathematics and Computer Science, Faculty of Arts and Sciences, Cankaya University, Ankara, 06530, Turkey. ${ }^{3}$ Department of Chemical and Materials Engineering, Faculty of Engineering, King Abdulaziz University, Jeddah, Saudi Arabia. ${ }^{4}$ Institute of Space Sciences, P.O. Box, MG-23, Magurele-Bucharest, R 76900, Romania. 


\section{Acknowledgements}

The authors sincerely thank the reviewers for their valuable comments that led to the present improved version of the original manuscript. And the first author wish to thank Dr.Y. Robinson, Director, RVS Technical Campus, Coimbatore - 641 402, Tamilnadu, India for his constant encouragements and support for this research work.

Received: 2 April 2013 Accepted: 29 June 2013 Published: 15 July 2013

\section{References}

1. Kilbas, A, Srivastava, H, Trujillo, Jj: Theory and Applications of Fractional Differential Equations. Elsevier, Amsterdam (2006)

2. Lakshmikantham, V, Leela, S, Vasundhara Devi, J: Theory of Fractional Dynamic Systems. Cambridge Scientific Publishers, Cambridge (2009)

3. Miller, KS, Ross, B: An Introduction to the Fractional Calculus and Fractional Differential Equations. Wiley, New York (1993)

4. Podlubny, I: Fractional Differential Equations. Academic Press, New York (1999)

5. Baleanu, D, Gunvenc, ZB, Machdo, JAT: New Trends in Nanotechnology and Fractional Calculus Applications. Springer, Berlin (2010)

6. Baleanu, D, Diethelm, K, Scalas, E, Trujillo, JJ: Fractional Calculus Models and Numerical Methods. Series on Complexity, Nonlinearity and Chaos. World Scientific, Singapore (2012)

7. Baleanu, D, Tenreiro Machado, JA, Luo, ACJ: Fractional Dynamics and Control. Springer, Berlin (2012)

8. $\mathrm{Wu}, \mathrm{GC}$, Baleanu, D: Variational iteration method for the Burgers' flow with fractional derivatives-new Lagrange multipliers. Appl. Math. Model. 37(9), 6183-6190 (2013)

9. Baleanu, D, Mustafa, OG, Agarwal, RP: On $L^{(p)}$ - solutions for a class of sequential fractional differential equations. Appl. Math. Comput. 218(5), 2074-2081 (2011)

10. Hernández, E, O'Regan, D, Balachandran, K: On recent developments in the theory of abstract differential equations with fractional derivatives. Nonlinear Anal. 73, 3462-3471 (2010)

11. Zhou, Y, Jiao, F: Existence of mild solutions for fractional neutral evolution equations. Comput. Math. Appl. 59, 1063-1077 (2010)

12. Zhou, Y, Jiao, F, Li, J: Existence and uniqueness for fractional neutral differential equations with infinite delay. Nonlinear Anal. 71, 3249-3256 (2009)

13. Baleanu, D, Mustafa, OG: On the global existence of solutions to a class of fractional differential equations. Comput. Math. Appl. 59(5), 1835-1841 (2010)

14. Baleanu, D, Mustafa, OG, Agarwal, RP: An existence result for a superlinear fractional differential equation. Appl. Math. Lett. 23(9), 1129-1132 (2010)

15. dos Santos, JPC, Arjunan, MM, Cuevas, C: Existence results for fractional neutral integro-differential equations with state dependent delay. Comput. Math. Appl. 62(3), 1275-1283 (2011)

16. dos Santos, JPC, Vijayakumar, V, Murugesu, R: Existence of mild solutions for nonlocal Cauchy problem for fractional neutral integro-differential equation with unbounded delay. Commun. Math. Anal. 14(1), 59-71 (2013)

17. Ji, S, Li, G, Wang, M: Controllability of impulsive differential systems with nonlocal conditions. Appl. Math. Comput. 217, 6981-6989 (2011)

18. Wang, JR, Fan, Z, Zhou, Y: Nonlocal controllability of semilinear dynamic systems with fractional derivative in Banach spaces. J. Optim. Theory Appl. 154(1), 292-302 (2012)

19. Pazy, A: Semigroups of Linear Operators and Applications to Partial Differential Equations. Springer, New York (1983)

20. Yan, B: Boundary value problems on the half-line with impulses and infinite delay. J. Math. Anal. Appl. 259(1), 94-114 (2001)

21. Kamenskii, M, Obukhovskii, V, Zecca, P: Condensing Multivalued Maps and Semilinear Differential Inclusions in Banach Spaces. de Gruyter, Berlin (2001)

22. O'Regan, D, Precup, R: Existence criteria for integral equations in Banach spaces. J. Inequal. Appl. 6, 77-97 (2001)

23. Mönch, H: Boundary value problems for nonlinear ordinary differential equations of second order in Banach spaces. Nonlinear Anal. 4, 985-999 (1980)

24. Banas, J, Goebel, K: Measure of Noncompactness in Banach Spaces. Lecture Notes in Pure and Applied Mathematics. Marcel Dekker, New York (1980) 